\title{
Mass Segregation and Tidal Tails of the Globular Cluster NGC 7492
}

\author{
Kang Hwan Lee \\ Astrophysical Research Center for the Structure and Evolution of the Cosmos, Sejong \\ University, Seoul 143-747, Korea \\ Centre for Astrophysics \& Planetary Science, School of Physical Sciences, University of \\ Kent, Canterbury, Kent CT2 7NR, UK \\ khlee@arcsec.sejong.ac.kr \\ Hyung Mok Lee \\ Astronomy Program, SEES, Seoul National University, Seoul 151-742, Korea \\ hmlee@astro.snu.ac.kr \\ Gregory G. Fahlman \\ Herzberg Institute of Astrophysics, 5071 West Saanich Road, Victoria, British Columbia \\ V9E 2E7, Canada \\ Greg.Fahlman@nrc-cnrc.gc.ca \\ and \\ Hwankyung Sung \\ Department of Astronomy \& Space Science, Sejong University, Seoul 143-747, Korea \\ sungh@arcsec.sejong.ac.kr
}

Received ; accepted 


\begin{abstract}
We present a wide field CCD photometric study of Galactic globular cluster NGC 7492. The derived $V R$ color-magnitude diagram extends down to about 3.5 mag below the cluster main sequence turn-off. The field covers $42^{\prime} \times 42^{\prime}$ about 3 times larger than the known tidal radius of this cluster. The sample of cluster member candidates obtained by CMD-mask process has been used to construct luminosity and mass functions and surface density map. NGC 7492 has a very flat mass function with very little variation in the slope with the distance from the cluster center. However, there is a clear evidence for the increase of the slope of the mass function from inner to outer regions, indicating a mass segregation of the cluster. The surface density map of NGC 7492 shows extensions toward the Galactic anticenter (northeast) and northwest from the cluster center. A comparison of luminosity function for stars in the tails with that for stars within the tidal radius suggests that the extensions shown in the surface density map could be a real feature. The overall shape of NGC 7492 is significantly flattened. If the flattened shape of the NGC 7492 is caused by its rotation, Galactic tidal field must have given important influences, since the initial rotation would have been almost completely removed by dynamical relaxation.
\end{abstract}

Subject headings: Galaxy: globular cluster: individual (NGC 7492) - stars: luminosity function, mass function 


\section{INTRODUCTION}

Globular clusters have attracted considerable attentions for the understanding of the process of Galaxy formation because they are thought to be fossil relics from the early formation history of the Galaxy. It is now well known that the globular clusters we see today may be only the survivors of an initial population. From the calculations of destruction rate, Lee \& Goodman (1995) and Gnedin \& Ostriker (1997) predicted that possibly as many as half of the present day Galactic globular clusters would be destroyed in the next Hubble time. Two body relaxation makes the velocity distribution of stars in a globular cluster toward a Maxwellian. As some stars acquire enough energy to escape from the cluster, the total mass slowly decreases (Spitzer \& Thuan 1972). The evaporation is also accelerated by the energy equipartition process which makes all stars have the same kinetic energy. It causes the mass segregation through which high-mass stars sink toward the central region of the cluster while low-mass stars have higher velocities and tend to occupy the outer region of the cluster (Spitzer 1987). Therefore mass segregation leads to the preferential loss of low-mass stars. In addition, clusters experience shocks when the external tidal field varies rapidly. Passage through the Galactic disk and close to the Galactic bulge also accelerate the destruction of clusters via gravitational shocks. Gnedin \& Ostriker (1997) found that tidal shocks contribute at least as much as two body relaxation to the destruction of the current globular clusters. The escaped stars may remain in the vicinity of the cluster for several Galactic orbits. As a result, the cluster is expected to have tidal tails.

Observational studies about tidal tails of globular clusters have been carried out only recently because of difficulties in wide field photometry. The signs of the existence of tidal tails around globular clusters were found in many previous studies using radial density profiles or two-dimensional density maps (Grillmair et al. 1995; Grillmair et al. 1996; Holland, Fahlman, \& Richer 1997; Lehmann \& Scholz 1997; Leon, Bergond, \& Vallenari 
1999; Leon, Meylan, \& Combes 2000; Testa et al. 2000; Odenkirchen et al. 2001; Sohn et al. 2003; Lee et al. 2003; Odenkirchen et al. 2003). The studies from radial density profiles suggest that many clusters have weak halo or tails of unbound stars which might result from tidal stripping. However, two-dimensional density maps obtained in these studies did not clearly confirm this suggestion because these are too complex and diffuse to be regarded as tidal tails.

More convincing evidences for the tidal tails came out of deep and wide CCD observations. Recently, using wide field photometric data from the Sloan Digital Sky Survey (SDSS), Odenkirchen et al. (2001, 2003) detected clear tidal tails around the Galactic globular cluster Palomar 5, and showed that this cluster is being tidally disrupted. The tails of Pal 5 extend over an arc of $10^{\circ}$ on the sky, corresponding to a projected length of 4 kpc at the distance of the cluster. They also suggested that the Pal 5 would be destroyed after the next disk crossing, which will happen in about $100 \mathrm{Myr}$. From the wide field photometry using CFH12K mosaic CCD, Lee et al. (2003) showed that the presence of an extra-tidal profile extending out to at least $\sim 30^{\prime}$ from the center of Galactic globular cluster M92. Using CFH12K wide field photometry, Sohn et al. (2003) found very weak tidal halos around the remote young globular clusters Pal 3 and Pal 4.

In this paper we study the mass segregation effect and spatial distribution of stars around the Galactic globular cluster NGC 7492 using the CHF12K VR photometry data. NGC 7492 is one of the most sparse globular clusters which is located far from the Galactic center and plane $\left(R_{G C}=24.9 \mathrm{kpc}, Z=-23.1 \mathrm{kpc}\right)$ and at $26.2 \mathrm{kpc}$ from the sun. The fundamental parameters of NGC 7492 are listed in Table 1. The first CCD photometry for this cluster was presented by Buonanno et al. (1987). They performed BV CCD photometry for NGC 7492 down to $V \sim 23$ roughly 2 mag fainter than the main-sequence turn-off. Côté, Richer \& Fahlman (1991, hearafter CRF91) carried out CCD photometry for this cluster 
covering a field of $2.2 \times 3.5$ containing cluster center. They presented a color-magnitude diagram (CMD) for NGC 7492 that traces the main sequence to about one magnitude fainter than that of Buonanno et al. (1987). They confirmed the cluster metallicity to be $[\mathrm{Fe} / \mathrm{H}]=-1.51$, and derived a distance modulus of $(m-M)_{0}=17.09 \pm 0.20 \mathrm{mag}$ by comparing the fiducial isochrone of NGC 6752 given in VandenBerg, Bolte, \& Stetson (1990). They also reported the discovery of 27 blue straggler candidates which are found to be more centrally concentrated than the cluster subgiants of similar brightness. If the blue stragglers are more massive than the stars at the main sequence turnoff, it would be the result of cluster mass segregation. They also presented star counts for ten annuli centered on the cluster core. However, they found no evidence for mass segregation in either the surface density profiles or in the luminosity functions at different radial positions. Possible reasons of failure in finding mass segregation are that the stellar mass range sampled by their data and/or the observed region is too small to find the change of luminosity function (LF) or mass function (MF). The small variation in the LF of NGC 7492 as a function of radius also can be expected from the study by Pryor, Smith, \& McClure (1986). Recently Leon, Meylan, \& Combes (2000) investigated the presence of tidal tails around 20 Galactic globular clusters including NGC 7492. Except for a tiny extension pointing toward the Galactic center, they did not find any other signs of tidal tail in NGC 7492. In this study, we present the investigation of mass segregation and tidal tails of NGC 7492 using deeper and wider field CCD photometry than that of CRF91.

\section{EDITOR: PLACE TABLE 1 HERE.}

In the following section, we present the observation and data reduction process. In section 3, we show CMDs of NGC 7492 and describe CMD-mask algorithm which select the sample of cluster member candidates. In section 4, we examine the mass segregation effect of the cluster using luminosity and mass functions of the cluster. In section 5, we 
examine the spatial distribution of stars around the cluster using surface density map and luminosity functions. Final results are summarized in section 6 .

\section{OBSERVATION AND DATA REDUCTION}

The observations were made with the $3.6 \mathrm{~m}$ Canada-France-Hawaii Telescope (CFHT) during 1999 Oct. 17-18 using CFH12K, a $6 \times 2$ mosaic of $2048 \times 4096$ CCDs. The CFH12K has an angular scale of $0 . " 206 /$ pixel at the $f / 4$ prime focus of the CFHT, giving a field of view of $42^{\prime} \times 28^{\prime}$. Each chip covers an area of $7^{\prime} \times 14^{\prime}$. Two adjacent fields were observed using $V$ and $R$ filters, covering an area of $42^{\prime} \times 42^{\prime}$, which extends beyond 3 times larger than the known tidal radius of this cluster, 8.'35 (Harris 1996). The positions of the observed fields relative to the center of NGC 7492 are shown in Figure 1, and observational information is given in Table 2. The points in Figure 1 represent the stars brighter than $V$ $=24$ and the point size is proportional to the brightness of stars. Each field was properly dithered to fill in the gaps between chips. We also obtained frames of fields containing Landolt (1992) standard stars for photometric calibration. All of the science images were

obtained under good seeing conditions (FWHM of $\sim 0 . " 8$ ). Several twilight flat-field, bias and dark frames were also taken.

\section{EDITOR: PLACE FIGURE 1 HERE.}

EDITOR: PLACE TABLE 2 HERE.

Pre-processing of the raw data, bias and dark subtraction and flat fielding, was done using the FITS Large Images Processing Software (FLIPS), a very efficient package for the reduction of CCD mosaic images developed by Jean-Charles Cuillandre at CFHT 
(Kalirai et al. 2001). FLIPS is designed to operate on individual chips within the CFH12K mosaic. First, the good exposures for each of the bias and dark exposures were median combined. For the flat-field images of each filter we averaged and sigma clipped the flats taken from observing run. Median combined bias and dark frames were then subtracted from the flat-field and object images. After the object images were debiased, the images were flat-fielded to account for pixel-to-pixel variations. FLIPS normalizes the background sky values to the chip with the highest sky level (lowest gain) and provides for a scaled data set with a smooth background on all chips. This makes the instrumental zero points for each chip almost equal. The statistics at various positions in the mosaic show that the flat-fielding to be typically better than $1 \%$ in both $V$ and $R$ filters.

Instrumental magnitudes of the point sources in the images were derived using the programs DAOPHOT II/ALLSTAR (Stetson 1994). For long exposure data (600s) we averaged the images of each filter using the ALIGN and IMCOMBRED commands within FLIPS. Short exposure images were used to get the instrumental magnitudes for stars saturated in long exposure images. The analysis is done separately for each chip because the point-spread function (PSF) is different for each of the 12 CCDs on the mosaic. We used a variable PSF to account for small changes in the profiles of stars over the large range of each CCD. We used the stellarity index of SExtractor (Bertin \& Arnouts 1996) for separating stars from background galaxies. As an example, in Figure 2 we show this parameter for sources detected in F1. Those objects with a stellarity index greater than 0.8 were considered to be stars. We applied a little more strict cut to avoid overestimation of faint stars which could exaggerate the mass segregation effect and tidal extensions of the cluster. This process also eliminates bad pixels caused by cosmic-ray hits. After further cuts, based on the two DAOPHOT parameters, the magnitude error $(<0.2)$ and the PSF fitting parameter, $\chi^{2}(<2)$, (we didn't consider sharpness parameter because we already used stellarity index) were made to eliminate spurious detections, the data sets in each filter 
were matched and the positions, instrumental magnitudes, and colors were obtained for all the stars.

\section{EDITOR: PLACE FIGURE 2 HERE.}

A number of Landolt (1992) standard stars in SA-92 were obtained during the observing run in order to convert the instrumental magnitudes to standard photometric magnitudes. The transformation equations to derive standard magnitudes are as follows;

$$
\begin{aligned}
& V=v+\alpha X+\beta(V-R)+Z_{v}, \\
& R=r+\alpha^{\prime} X+\beta^{\prime}(V-R)+Z_{r} .
\end{aligned}
$$

In these equations $v$ and $r$ are instrumental magnitudes and $\alpha, \alpha^{\prime}$ are the extinction coefficients of the airmass term $X, \beta, \beta^{\prime}$ are the transformation coefficient of the color term $(V-R)$, and $Z_{v}, Z_{r}$ are the zero-point shift for the $V$ and $R$ bands. Since we could get only $1 \sim 3$ standard stars on each chip, we applied the standard values for the color term and the atmospheric extinction coefficient given on the CFHT home page. Separate calibrations are actually required for each chip, because there are systematic differences between chips. However, the color term does not significantly change with different exposures or nights of observations and the extinction coefficient is very stable at CFHT site (Kalirai et al. 2001). We used standard stars to get the zero points of each chip which are the most critical part of the transformation equations. The instrumental zero points for the chips were almost identical because FLIPS normalizes the background sky value. The mean value of the differences between our photometry of the Landolt (1992) standard stars and the standard values is 0.01 . To check the accuracy of photometry and calibration we used stars in the dithered frames in each field and overlapped region between the two fields. The same stars in the different chips and at different locations in the field of view of the instrument 
showed differences less than limiting magnitude error (0.2) in all chips. Figure 3 shows the magnitude differences between the same stars in the different chips.

\section{EDITOR: PLACE FIGURE 3 HERE.}

\section{COLOR-MAGNITUDE DIAGRAM}

The derived CMD based on the long and short exposure data for the entire observed

region of the cluster is shown in Figure 4. Well-defined cluster sequence as well as field stars scattered over the entire CMD can be easily seen. In Figure 4, we can see a well-defined blue horizontal branch (BHB), and the absence of a red horizontal branch (RHB). Also

clearly visible are the red giant branch (RGB), subgiant branch (SGB), the main sequence (MS) turnoff at $V \sim 21 \mathrm{mag}$, and a MS that is extended down to about $V \sim 24.5 \mathrm{mag}$ which is roughly 3.5 mag fainter than turnoff. The characteristics of CMD morphology are described by CRF91 in detail. Since our main concern is the distribution of cluster stars, the contamination from field stars needs to be removed.

\section{EDITOR: PLACE FIGURE 4 HERE.}

To select cluster stars, we used the technique of the CMD-mask algorithm, which was introduced by Grillmair et al. (1995). In this way we can differentiate cluster members from the background and foreground field stars by means of comparing stars inside the tidal radius of the cluster with the field stars in a distant part of the field. The filtering mask is empirically chosen so as to optimize the ratio of cluster stars to field stars in the relatively sparsely populated outer region of the cluster. 
We regarded the regions beyond 2.5 times of tidal radius $\left(r>20 .^{\prime} 9\right)$ as background regions which is shown in Figure 1. In Figure 5, we show CMDs of the cluster region inside the tidal $\operatorname{radius}\left(r<r_{t}=8 .^{\prime} 35\right)$, and outer region $\left(r>2.5 \times r_{t}=20 .^{\prime} 9\right)$. The procedure was performed in the region $-0.6<V-R<0.9$ and $15<V<25.5$ which is indicated by rectangular boxes in Figure 5. The region was subdivided into a $30 \times 55$ array in which the individual subgrid elements were 0.05 mag wide in $V-R$ and 0.2 mag high in $V$.

\section{EDITOR: PLACE FIGURE 5 HERE.}

Assuming that the color-magnitude distribution of the field stars is constant across the observed field, a color-magnitude sequence for the cluster can be estimated from (Grillmair et al. 1995):

$$
f_{c l}(i, j)=n_{c l}(i, j)-g n_{f}(i, j)
$$

where $n_{c l}(i, j)$ and $n_{f}(i, j)$ refer to the number of stars with color index $i$ and magnitude index $j$, counted in an area of circle with radius $r<r_{t}$ around the cluster center, and around the background field region, respectively. The factor $g$ is the ratio of the area of the cluster region to that of the background field region. The signal-to-noise ratio of the expected true number of cluster stars for each subarea was computed from:

$$
s(i, j)=\frac{f_{c l}(i, j)}{\sqrt{n_{c l}(i, j)+g^{2} n_{f}(i, j)}} .
$$

From the signal-to-noise $s$ we obtain a filtering mask by isolating the region in the color-magnitude subgrid with $s>s_{\text {lim }}$. To select the optimal range of color and magnitude, the elements of $s(i, j)$ were sorted into a gradually descending order over the one-dimensional index $l$ first. From the subgrid element with the highest $s$ value, cumulative star counts were carried out in the cluster region using progressively larger areas of the CMD, $a_{k}=k a_{l}$, where $a_{l}=0.016 \mathrm{mag}^{2}$ is the area of a single element in the color-magnitude array. Then 
the cumulative signal-to-noise ratio, $S\left(a_{k}\right)$, can be computed from:

$$
S\left(a_{k}\right)=\frac{N_{c l}\left(a_{k}\right)-g N_{f}\left(a_{k}\right)}{\sqrt{N_{c l}\left(a_{k}\right)+g^{2} N_{f}\left(a_{k}\right)}},
$$

where $N_{c l}\left(a_{k}\right)$ and $N_{f}\left(a_{k}\right)$ are the cumulative number of stars in the corresponding subarea in the cluster region and in the outer background field region, respectively. Now, $n_{c l}(l)$ and $n_{f}(l)$ refer to the number of stars within the cluster region and background field region, having ordered color-magnitude index $l$. This cumulative function reaches a maximum for

a particular subarea of the CMD plane. Based on the peak value of $S$, a threshold value of $s_{\text {lim }}$ is determined. The heavy lines in Figure 5 indicate the filtering mask differentiating cluster members from the field stars. By extracting stars outside the filtering mask in the CMD, the field contamination was reduced by a factor of $\sim 4$.

\section{MASS SEGREGATION}

It is well known that the radial variation of luminosity functions (LFs) and mass functions (MFs) give a crucial hint of the mass segregation effect in a globular cluster. LFs were constructed using all CMD-selected stars within the cluster's tidal radius. To build a reliable LF especially in the crowded field, incompleteness corrections must be applied correctly. To correct for the incompleteness of our photometry, we ran artificial star tests on the $V$ frames, using ADDSTAR routine in IRAF/DAOPHOT. First, we added artificial stars in each $0.5 \mathrm{mag}$ bin randomly on all original images. The number of added stars was designed not to exceed $10 \%$ of the total number of stars that is actually present in that bin so as not to enhance original crowding. The new frames obtained in this way were then reduced in an identical manner used on the original frame. Figure 6 shows a sample of the plots of the differences between input and output magnitudes for recovered artificial stars selected by the same criteria applied to original image reduction. Since most of values do not exceed $0.3 \mathrm{mag}$, the stars which have magnitude differences smaller than $0.3 \mathrm{mag}$ 
are considered as recovered stars. We repeated these procedures 10 times on each chip to obtain meaningful statistics in each magnitude bin. Finally, the incompleteness correction factor $f$ is obtained by $f=n_{r e c} / n_{\text {add }}$, where $n_{\text {add }}$ is the number of added stars and $n_{\text {rec }}$ is the number of recovered stars. To examine the accuracy of the incompleteness correction, we ran artificial star tests in another way. We selected two chips in each field of $V$ and $R$ frames. Then the artificial stars which have similar color band of the cluster MS and RGB stars have been added on the $V$ and $R$ images with the same method described above. The new frames were then reduced in an identical manner used on the original frame. The stars, which have magnitude differences smaller than 0.3 mag and color differences smaller than $0.2 \mathrm{mag}$ are considered as recovered stars. The incompleteness correction factors obtained from this way were in accord with the previous results within $5 \%$ on the same chips. The incompleteness correction factors depended on the distance from the cluster center and did not show any differences between chips. The resulting incompleteness correction factors were then applied to the raw LFs. To investigate any spatial variation of LF, the LFs for the inner region $\left(0^{\prime}<r<1 .^{\prime} 3\right)$ and the outer region $\left(1 .^{\prime} 3<r<8 .^{\prime} 35\right)$ are constructed separately. The regions are divided to contain similar number of stars in each region. The corrected number of stars in each magnitude bin and the incompleteness factors are given in Table 3, and the corrected LFs are plotted in Figure 7. The histogram in Figure 7 is the LF for all selected stars within tidal radius $\left(r<8 .^{\prime} 35\right)$. The solid line overlaid on the inner LF is that of NGC 7492 for $0^{\prime}<r<2^{\prime}$ derived by CRF91, which agrees very well with ours. This agreement implies that the incompleteness corrections are properly applied.

\section{EDITOR: PLACE FIGURE 6 HERE.}

EDITOR: PLACE TABLE 3 HERE. 
The presence of mass segregation be seen from the radial variation of LFs. The slope of LFs of globular clusters are known to increase toward the outer parts of the cluster where low mass stars preferentially occupy as a result of mass segregation. However, we could not find any definite evidence from Figure 7 alone. As discussed below, the small variation in the LF of NGC 7492 as a function of radius is not an unexpected result. The LFs were converted into mass functions (MFs) using the mass-luminosity relation of Baraffe et al. (1997) and a distance modulus of $(m-M)_{0}=17.09$ (CRF91). The calculated MFs are shown in Figure 8. Due to the small number of stars brighter than the MS turn-off, only stars with $V>20$ mag have been used. Filled circles represent the MF for the inner region $\left(0^{\prime}<r<1 .^{\prime} 3\right)$ while open circles for outer region $\left(1 .^{\prime} 3<r<8 .^{\prime} 35\right)$. Open triangles represent the MF derived by CRF91. Because of our LF extends only to $M_{V} \sim 7.5$ the MF is defined only in the range $0.6 \sim 0.8 M_{\odot}$. For this reason, it is difficult to say anything definitely about the slope of the main sequence MF based solely on Figure 8. CRF91 derived a slope of the MF $x=-1.1 \pm 0.5$ using the theoretical LF from Drukier et al. (1988). Here $x$ represents the slope of a MF of the form

$$
\Phi(M) d M \propto M^{-(1+x)} d M
$$

We insert lines in Figure 8 which show several power law indices covering the slope from CRF91. Although it is difficult to fit a single power law to MFs of this cluster, we can conclude that the $x$ value lies within the range from previous study. It is noteworthy that the cluster has a very flat MF and the variation of the MFs from inner to outer regions is very small. Although the slope changes by very small amount, we can see a clear tendency of increase in the slope of MF from inner to outer regions in Figure 8. It could be a sign of mass segregation in this cluster.

\section{EDITOR: PLACE FIGURE 7 HERE.}




\section{EDITOR: PLACE FIGURE 8 HERE.}

Small change in the MF slope does not mean that this cluster is not fully relaxed. Pryor, Smith \& McClure (1986) have investigated the effects of mass segregation on main sequence MFs for globular clusters with various central concentrations. They found that mass segregation depends on core concentration parameter $(c)$ and the slope of mass functions $(x)$. They showed that the mass segregation effect appears more clearly in models with large concentrations and steep (larger $x$ ) mass functions. For a fully relaxed cluster having $c=1.0$ and $x \sim-1$, there will be very little change in the MFs due to mass segregation. Using the HST photometry, Grillmair \& Smith (2001) investigated the main-sequence LF of Pal 5. According to their results, Pal 5 has a very flat MF $(x=-0.5)$ and shows very little evidence for mass differentiation between the core of the cluster and the half-mass radius. They suggested that Pal 5 has lost a large fraction of its original stellar content as a result of tidal shocking. As mentioned in section 1, Pal 5 appears to be in the final phase of tidal disruption (Odenkirchen et al. 2003). In any globular cluster the form of the present day mass function depends on the initial mass function and the process of dynamical evolution of the cluster. McClure et al. (1986) suggested that the slope of MF is related with metallicity of cluster. They proposed that the observed strong dependence of MF slopes on metallicity reflects, at least in part, properties of the initial mass functions with which the clusters formed. Later, Djorgovski, Piotto, \& Capaccioli (1993) demonstrated that the MF slopes are determined not only by the metallicity but also by the location in the Galaxy. They showed that the clusters closer to the Galactic center have flatter MFs. At a given Galactocentric distance $\left(R_{G C}\right)$, clusters with a smaller distance from the Galactic plane $(Z)$ have flatter MFs, and at a given position, clusters with lower metallicity have steeper MFs. From N-body simulations, Capaccioli, Piotto, \& Stiavelli (1993) interpreted the dependence on position as the effect of tidal shocks. Disk 
and bulge shocking acts preferentially on stars located in the outer region of the cluster and leads to a loss of low mass stars. As a result the clusters near the Galactic center and plane, tend to have flatter MFs. Since NGC 7492 has intermediate metallicity $([\mathrm{Fe} / \mathrm{H}]=$ -1.51) among the Galactic globular clusters and large distant from Galactic center and plane $\left(R_{G C}=24.9 \mathrm{kpc} ; Z=-23.1 \mathrm{kpc}\right)$, flat MF of NGC 7492 can not be interpreted neither by its metallicity nor by Galactic position. However, dynamical evolution of a globular cluster can not be determined only by present position but also by cluster's orbit. Piotto, Cool, \& King (1997) presented a comparison of deep HST LFs of four Galactic globular clusters. They showed that three of four clusters (M15, M30, and M92) have nearly identical LFs, whereas NGC 6397 has a distinctly different LF, especially in the fainter part. They suggested that the three globular clusters which have similar LFs were formed with similar MFs and suffered very little changes (or experienced similar changes regardless of the location), and that the deficiency of low-mass stars in NGC 6397 was due to tidal shocks, stellar evaporation through internal relaxation, or a combination of the two. Relatively flat MF of NGC 6397 could be explained by its orbit given by Dauphle et al. (1996) which is very vulnerable to tidal shocks. Later, comparing MF slopes for 7 Galactic globular clusters Piotto \& Zoccali (1999) suggested that the flattening of MF slopes might be related to the cluster's dynamical evolution. From the N-body simulations, Baumgardt \& Makino (2003) predicted that the slope of MFs of the clusters should decrease constantly as the clusters evolve, and there is a good correlation between the slopes and the cluster lifetimes. If the flat MF of NGC 7492 is not primordial it might be a result of dynamical evolution of this cluster. When the Galactic orbit of NGC 7492 is determined the influence of Galactic tidal shocks on this cluster could be known. 


\section{SPATIAL DISTRIBUTION OF STARS}

\subsection{Surface Density Map}

In order to characterize the distribution of stars around the cluster, we constructed surface number density map. The sample of cluster member candidates obtained by CMD-mask process in the previous section was used to construct surface density map. Initially, the candidate cluster stars in the surveyed region were binned on a grid of $0 . ' 2 \times 0 .{ }^{\prime} 2$. Unfortunately, our surveyed region is not wide enough to get proper background maps. However, we used field stars having color greater than 0.9 to check the gradient of distribution of field stars and possible photometric biases in the entire surveyed region. After constructing the map of field stars by binning $1^{\prime} \times 1^{\prime}$, we smoothed it to get a surface map dominated only by the smooth gradient of field stars. We subtracted this field star map from the CMD-selected one. We then convolved the map with a Gaussian kernel of width 1.'0. The resulting smoothed surface density map is shown in Figure 9.

\section{EDITOR: PLACE FIGURE 9 HERE.}

We overlaid contour levels and marked the tidal radius with a thick circle. The arrow indicates the direction to the galactic center. We can see a marginal extension toward the Galactic anticenter (northeast) in Figure 9. Also there seems to be a small extension to the northwest from the cluster center. Many N-body simulations of globular cluster tidal tails show that the stars evaporated from the cluster form a twisted, two-sided lobe distribution of cluster member stars (Combes, Leon, \& Meylan 1999; Yim \& Lee 2002; Lee et al. 2004). However, the shape of such lobes depends on the orientation of the cluster's orbit to our line of sight. Combes, Leon, \& Meylan (1999) showed that the lobes can be asymmetric and even seen one-sided according to the cluster's orbits and projection effects in their Figure 13 and Figure 15. The extensions shown in Figure 9 can not be regarded as a real feature 
solely based on the surface density map. We will check the significance of the extensions using luminosity functions in the following subsection.

From Figure 9, we can see that the cluster NGC 7492 has very flattend shape. White \& Shawl (1987) have measured the projected axial ratio $(b / a)$ of 100 Galactic globular clusters, where $a$ and $b$ denote semi-major and semi-minor radii, respectively. They obtained the value of $\langle b / a\rangle=0.76$ from NGC 7492 while mean axial ratio was $\langle b / a\rangle=0.93 \pm 0.01$. We used ELLIPSE task in IRAF/STSDAS to fit the elliptical shape of the cluster. Figure 10 shows the axial ratio of the cluster as a function of the semi-major axes of the ellipses. The axial ratio decreased and reached to a value of 0.77. White \& Shawl (1987) argued that the flattened shape of the globular clusters can be caused by either anisotropy in velocity dispersion or rotation. The tidally truncated clusters tends to become quickly isotropic (Takahashi \& Lee 2000), the flattening is likely due to rotation (Combes, Leon \& Meylan 1999). Kinematical data also showed that the flattening could be explained by rotation, and that the minor axes are nearly coincident with the rotation axes (Meylan \& Mayor 1986). The initial angular momentum of a cluster is expected to disappear with time by the escape of stars carrying some angular momentum. Kim et al. (2002) showed using the Fokker-Plank method that the initial rotation decreases with time after core-collapse and finally disappear. As a result, the flattening of a globular cluster could be related with its dynamical age. From a compilation of data on globular cluster flattenings in our Galaxy and M31, Davoust \& Prugniel (1990) showed that globular clusters with shorter relaxation times tend to be rounder. They suggested a scenario which globular clusters are initially flattened and become rounder as they lose stars and angular momentum. According to this scenario, NGC 7492 would be the one which has the youngest dynamical age among the Galactic globular clusters. However considering the flat MF of NGC 7492, it is unlikely that this cluster maintain initial dynamical status. If the flattening of NGC 7492 cannot be explained only by initial rotation of this cluster, we should consider some alternative 
sources of globular cluster rotation. One possible alternative is tidal interaction with the Galaxy. From N-body simulations Lee et al. (2004) showed that the globular clusters gain angular momentum from tidal interactions with host galaxy. If a cluster rotates by the tidal interaction with the galaxy, the orientation of the rotation axis would be the same as its revolution axis. When we get the orbit of NGC 7492, the influence of tidal interaction on its rotation could be examined.

\section{EDITOR: PLACE FIGURE 10 HERE.}

\subsection{Luminosity Functions}

Figure 9 shows marginal extensions of stellar distributions around NGC 7492 toward northeast and northwest from the cluster center. However we can not regard the extensions shown in Figure 9 as a real feature using only the surface density map. We therefore analyzed the LFs of the stars around the clusters to check the significance of the extensions.

In figure 11, we show the cumulative LF for stars (solid line) located in outer northern part $\left(r>r_{t}\right)$ where extensions appear. The dashed line indicates the LF for stars within the tidal radius $\left(r_{t}\right)$ of NGC 7492 . For comparison, we also show the LF for stars in southern part of the cluster as a dotted line. These LFs are constructed using CMD-selected stars. Incompleteness corrections are properly applied to each LF as described in the previous section. The LFs are normalized by the total number of stars for each selected group. A Kolmogorov-Smirnov (K-S) test of the LFs for stars in the outer northern part (solid line) compared to that for stars within the tidal radius (dashed line) gives a significance level of $75 \%$ probability that both cumulative LFs follow the same distribution. On the other hand, the K-S significance level of cumulative LF for stars in the southern part (dotted line) is less than $1 \%$. Since mass segregation effect of this cluster is very small, we can 
expect that the LF for stars in the tidal tails of the cluster would be almost the same with that for stars within the tidal radius in the limit of our analysis (Grillmair \& Smith 2001). Odenkirchen (2003) also showed that the LF for the stars in the tidal tails of Pal 5 is in very good agreement with the LF in the cluster. Theses indicate that the extensions shown in the surface density map (Figure 9) could be a real feature.

\section{EDITOR: PLACE FIGURE 11 HERE.}

\section{SUMMARY}

We have carried out a wide field CCD photometry of NGC 7492 and investigated the dynamical structure of the cluster. The observations with the CFH12K mosaic CCD cover the area of $42^{\prime} \times 42^{\prime}$, about 3 times larger than the known tidal radius of this cluster. We used the technique of the CMD-mask algorithm to select cluster member star candidates, and we have used these stars to examine the characteristics of the spatial distribution of stars around the clusters. The mass segregation effect and stellar distribution around the cluster have been investigated by means of a completeness-corrected LFs and MFs and the surface density map.

The slope of cluster main sequence MF slightly increases toward the outer part of the cluster, as expected from mass segregation. It could be a verification of mass segregation effect of this cluster which was suggested doubtfully only from the distribution of blue stragglers by CRF91. Although the change in the MF slope is very small it does not mean that this cluster is not fully relaxed. The relatively flat MF slope of NGC 7492 and its small mass might be a result of dynamical evolution of this cluster.

Surface density map shows possible extensions of spatial stellar distributions beyond 
the tidal radius of NGC 7492. The extensions of the tails of NGC 7492 are oriented toward the Galactic anticenter (northeast) and northwest from the cluster center. A comparison of LF for stars in the tails with that for stars within the tidal radius suggests that the extensions shown in the surface density map could be a real feature. Surface density map also shows that the NGC 7492 has very flat shape. The axial ratio $(b / a)$ of this cluster decrease with semi-major axis and reached to a value of 0.77 which is one of the smallest values the Galactic globular clusters have. If the flattened shape of this cluster is caused by its rotation, it might have been much affected by Galactic tidal field.

Pryor et al. (1991) argued that low concentration (low-c) clusters lost much of their original stellar mass over extended periods of time through the evaporation and stripping of stars. Recently, Odenkirchen et al. (2003) showed clear evidences that the low-mass, low-c Galactic halo cluster Pal 5 is being tidally disrupted. In addition to previous studies, flat mass function, tidal extensions, and flat shape of the cluster which are investigated in this study suggest that NGC 7492 might have experienced a lot of dynamical processes through tidal interaction with the Galaxy. However, kinematic data of NGC 7492 are not obtained yet. The information on the orbits of individual halo clusters can produce powerful constraints on the Galactic potential. We need spectroscopic observations and proper motion studies about NGC 7492 to get the information about the rotation and the Galactic orbit of the cluster. We also encourage deeper and wider CCD photometry of this cluster to find possible tidal tails extending over the coverage of this study.

HML was supported by the KOSEF grant No. R01-1998-00023. H.S. acknowledges the support of the Korea Science and Engineering Foundation (KOSEF) to the Astrophysical Research Center for the Structure and Evolution of the Cosmos (ARCSEC') at Sejong University. 


\section{REFERENCES}

Baraffe, I., Chabrier, G., Allard ,F., \& Hauschildt, P. H., 1997, A\&A, 327, 1054

Baumgardt, H., \& Makino, J., 2003, MNRAS, 340, 227

Bertin, E., \& Arnouts, S., 1996,A\&AS, 117, 393

Buonanno, R., Corsi, C. E., Ferraro, I., \& Fusci Pecci, F., 1987, A\&AS, 67, 327

Capaccioli, M., Piotto, G., \& Stiavelli, M., 1993, MNRAS, 261, 819

Combes F., Leon S., \& Meylan G., 1999, A\&A, 352, 149

Côté, P., Richer, H. B., \& Fahlman, G. G., 1991, AJ,102,

Dauphole, B., Geffert, M., Colin, J., Ducourant, C., Odenkirchen, M., \& Tucholke, H. -J., 1996, A\&A, 304, 202

Davoust, E., \& Prugniel, P., 1990, A\&A, 230, 67

Djorgovski, S., Piotto, G., \& Capaccioli, M., 1993, AJ, 105, 2148

Drukier, G. A., Fahlman, G. G., Richer, H. B., \& VandenBerg, D. A., 1988, ApJ, 95, 1415

Gnedin, O. Y., \& Ostriker, J. P., 1997, ApJ, 474, 223

Grillmair, C. J., Freeman, K. C., Irwin, M., \& Quinn, P. J., 1995, AJ, 109, 2553

Grillmair, C. J., Ajhar, E. A., Faber, S. M., Baum, W. A., Holtzman, J. A., Lauer, T. R., Lynds, C. R., \& O’Neil, E. J, 1996, AJ, 111, 2293

Grillmair, C. J., \& Smith, G. H., 2001, AJ, 122, 3231

Harris W. E., 1996, AJ, 112, 1487 
Holland S., Fahlman G. G., \& Richer H. B., 1997, AJ, 114, 1488

Kalirai, J. S., Richer, H. B., Fahlman, G. G., Cuillandre, J.-C., Ventura, P., D’Antona, F., Bertin, E., Marconi, G., \& Durrell, P. R., 2001, AJ, 122, 257

Kim, E., Einsel, C., Lee, H. M., Spurzem, R., \& Lee, M. G. 2002, MNRAS, 334, 310

Landolt, A. U., 1992, AJ, 104, 340

Lee, H. M., \& Googman, G., 1995, ApJ, 443, 109

Lee, K. H., Lee, H. M., Fahlman, G. G., \& Lee, M. G., 2003, AJ, 126, 815

Lee, K. H., Lee, H. M., Yim, K. J., \& Sung, H., 2004, in preparation

Lehmann I., \& Scholz R. -D., 1997, A\&A, 320, 776

Leon, S., Bergond, G., \& Vallenari, A., 1999, A\&A, 344, 450

Leon, S., Meylan, G., \& Combes, F. 2000, A\&A, 359, 907

McClure, R. D., VandenBerg, D. A., Smith, G. H., Fahlman, G. G., Richer, H. B., Hesser, J. E., Harris, W. E., Stetson, P. B., \& Bell, R. A., 1986, ApJ, 307, L49

Meylan, G., \& Meyor, M., 1986, A\&A, 166, 122

Odenkirchen M., Grebel E. K., Rockosi C. M., Dehnen W., Ibata R., Rix H. W., Stolte A., Wolf C., Anderson, Jr. J. E., Bahcall N. A., Brinkmann J., Csabai I.,Hennessy G., Hindsley R. B., Ivezic Z., Lupton R. H., Munn J. A., Pier J. R., Stoughton C., \& York D. G., 2001, ApJ, 548, L165

Odenkirchen, M., Grebel, E. K., Dehnen, W., Rix, H.-W., Yanny, B., Newberg, H. J., Rockosi, C. M., Martinez-Delgado, D., Brinkmann, J., \& Pier, J. R., 2003, AJ, 126, 2385 
Piotto, G., Cool, A. M., \& King, I. R., 1997, AJ, 113, 1345

Piotto, G., \& Zoccali, M., 1999, A\&A, 345, 485

Pryor, C., Smith, G. H., \& McClure, R. D., 1986, AJ, 92, 1358

Pryor, C., McClure, R. D., Fletcher, J. M., \& Hesser, J. E., 1991, AJ, 102, 1026

Sohn, Y.-J, Park, J.-H., Rey, S.-C., Kim, H.-I., Oh, S. J., Lee, S.-G., Lee, M. G., \& Han, W., 2003, AJ, 126, 803

Spitzer, L., 1987, Dynamical Evolution of Globular Clusters (Princeton: Princeton Univ. Press)

Spitzer, L., \& Thuan, T. X., 1972, ApJ, 175, 31

Stetson, P. B., 1994, PASP, 106, 250

Takahashi, K., \& Lee, H. M., 2000, MNRAS, 316, 671

Testa V., Zaggia S. R., Andreon G. L., Scaramella R., Djorgovski S. G.,\& de Carvalho R., 2000, A\&A, 356, 127

VandenBerg, D. A., Bolte, M., \& Stetson, P. B., 1990, AJ, 100, 445

White, R. E., \& Shawl, S. J., 1987, ApJ, 317, 246

Yim, K. J., \& Lee, H. M., 2002, J. Korean Astron. Soc., 35, 75 
Fig. 1.- Location of 2 observed fields, with the origin set on the cluster center. The circles indicate known tidal radius $r_{t}=8 .^{\prime} 35$ (inner) and 2.5 times of tidal radius $\left(r=20 .^{\prime} 9\right)$. Each frame has a field of view of $42^{\prime} \times 28^{\prime}$. The points represent the stars brighter than $V=24$. The point size is proportional to the brightness of stars.

Fig. 2.- The stellarity index of objects in F1 region. Sources with a stellarity index greater than 0.8 are considered to be stars.

Fig. 3.- The differences between the same stars in the different chips.

Fig. 4. - CMD for all calibrated stars. This is the result of merging the long and short exposure data.

Fig. 5. - CMDs for the stars within the tidal radius $\left(r<r_{t}=8 .^{\prime} 35\right)$, and outer region $\left(r>20 .^{\prime} 9\right)$. The heavy lines indicate criteria for selecting cluster member candidates to reduce field star contribution.

Fig. 6.- The result of artificial star test for one direction from cluster center. The difference $\Delta \mathrm{V}$ is $V_{\text {in }}-V_{\text {out }}$.

Fig. 7.- Luminosity functions of inner $\left(0^{\prime}<r<1 .^{\prime} 3\right)$ and outer $\left(1 .^{\prime} 3<r<8 .^{\prime} 35\right)$ region for NGC 7492. The histogram is the LF for all stars within $r<8 .^{\prime} 35$. A solid line overlayed with inner LF is the result from Côté, Richer \& Fahlman (1991). For convenient comparison, the LFs are arbitrarily shifted.

Fig. 8.- Mass functions of inner $\left(0^{\prime}<r<1 .^{\prime} 3\right)$ and outer $\left(1 .^{\prime} 3<r<8 .^{\prime} 35\right)$ region for NGC 7492. Filled circles represent the MF for the inner region while open circles for outer region. Open triangles in the upper panel are the result from CRF91. The solid lines represent several power law indices.

Fig. 9.- Surface density map and contours of levels of and all selected stars. The tidal 
radius is marked as a thick circle. The arrow indicates the direction of the Galactic center. Contours are drawn at 1,2, 3 and $5 \sigma$ of the background.

Fig. 10. - The axial ratio $(b / a)$ of the cluster NGC 7492 as a function of the length of semi-major radius.

Fig. 11.- The cumulative LFs for stars located in the northern part where tidal extensions are shown (solid line) and within the tidal radius (dashed line) of the cluster NGC 7492. Dotted line indicates the LF for southern part of outer region of the cluster. 
Table 1. Coordinates and Physical Parameters for NGC 7492

\begin{tabular}{lrr}
\hline \hline \multicolumn{1}{c}{ Parameter } & \multicolumn{1}{c}{ Value } & Reference \\
\hline$\alpha(2000)$ & $23^{h} 08^{m} 26.7^{s}$ & Harris (1996) \\
$\delta(2000)$ & $-15^{\circ} 36^{\prime} 41^{\prime \prime}$ & Harris (1996) \\
$l(2000)(\mathrm{deg})$ & 53.39 & Harris $(1996)$ \\
$b(2000)(\mathrm{deg})$ & -63.48 & Harris $(1996)$ \\
$\log \rho_{0}\left(M_{\odot} / \mathrm{pc}^{3}\right)$ & 0.97 & Harris $(1996)$ \\
$r_{t}(\operatorname{arcmin})$ & 8.35 & Harris $(1996)$ \\
$c=\log \left(r_{t} / r_{c}\right)$ & 1.00 & Harris $(1996)$ \\
{$[\mathrm{Fe} / \mathrm{H}]$} & -1.51 & CRF91 \\
$(m-M)_{0}$ & 17.09 & CRF91 \\
$E(B-V)$ & 0.00 & CRF91 \\
\hline
\end{tabular}


Table 2. Obsevational Information of NGC 7492

\begin{tabular}{lcccc}
\hline \hline Field & $V$-Filter & date & $R$-Filter & date \\
\hline F1 & $2 \times 20 \mathrm{~s}$ & Oct. 171999 & $1 \times 20 \mathrm{~s}$ & Oct. 171999 \\
F1 & $1 \times 100 \mathrm{~s}$ & Oct. 171999 & $1 \times 100 \mathrm{~s}$ & Oct. 171999 \\
F1 & $6 \times 600 \mathrm{~s}$ & Oct. 171999 & $6 \times 600 \mathrm{~s}$ & Oct. 171999 \\
F2 & $1 \times 20 \mathrm{~s}$ & Oct. 181999 & $1 \times 20 \mathrm{~s}$ & Oct. 181999 \\
F2 & $1 \times 100 \mathrm{~s}$ & Oct. 181999 & $1 \times 100 \mathrm{~s}$ & Oct. 181999 \\
F2 & $4 \times 600 \mathrm{~s}$ & Oct. 181999 & $4 \times 600 \mathrm{~s}$ & Oct. 181999 \\
\hline
\end{tabular}


Table 3. Corrected Luminosity Functions and Incompleteness Correction Factors

\begin{tabular}{|c|c|c|c|c|c|c|}
\hline \multirow[t]{2}{*}{$\mathrm{V}$} & \multicolumn{2}{|c|}{ Inner $\left(0^{\prime}<r<1 .^{\prime} 3\right)$} & \multicolumn{2}{|c|}{ Outer $\left(1 .^{\prime} 3<r<8^{\prime} .35\right)$} & \multicolumn{2}{|c|}{ Global } \\
\hline & $\mathrm{N}$ & $f$ & $\mathrm{~N}$ & $f$ & $\mathrm{~N}$ & $f$ \\
\hline $15.5-16.5$ & 4.0 & 1.0 & 3.0 & 1.0 & 7.0 & 1.0 \\
\hline $16.5-17.5$ & 10.0 & 1.0 & 12.0 & 1.0 & 22.0 & 1.0 \\
\hline $17.5-18.5$ & 32.0 & 1.0 & 26.0 & 1.0 & 58.0 & 1.0 \\
\hline $18.5-19.5$ & 28.0 & 1.0 & 22.0 & 1.0 & 50.0 & 1.0 \\
\hline $19.5-20.5$ & 73.2 & 0.99 & 49.5 & 0.99 & 122.7 & 0.98 \\
\hline $20.5-21.5$ & 490.0 & 0.90 & 310.4 & 0.96 & 800.4 & 0.94 \\
\hline $21.5-22.5$ & 963.3 & 0.79 & 669.2 & 0.91 & 1632.5 & 0.87 \\
\hline $22.5-23.5$ & 1263.8 & 0.69 & 943.3 & 0.90 & 2207.1 & 0.80 \\
\hline $23.5-24.5$ & 1274.4 & 0.43 & 1068.6 & 0.70 & 2343.0 & 0.58 \\
\hline
\end{tabular}


This figure "Lee.fig01.jpg" is available in "jpg" format from: http://arxiv.org/ps/astro-ph/0409037v1 
This figure "Lee.fig02.jpg" is available in "jpg" format from: http://arxiv.org/ps/astro-ph/0409037v1 
This figure "Lee.fig03.jpg" is available in "jpg" format from: http://arxiv.org/ps/astro-ph/0409037v1 
This figure "Lee.fig04.jpg" is available in "jpg" format from: http://arxiv.org/ps/astro-ph/0409037v1 
This figure "Lee.fig05.jpg" is available in "jpg" format from: http://arxiv.org/ps/astro-ph/0409037v1 
This figure "Lee.fig06.jpg" is available in "jpg" format from: http://arxiv.org/ps/astro-ph/0409037v1 

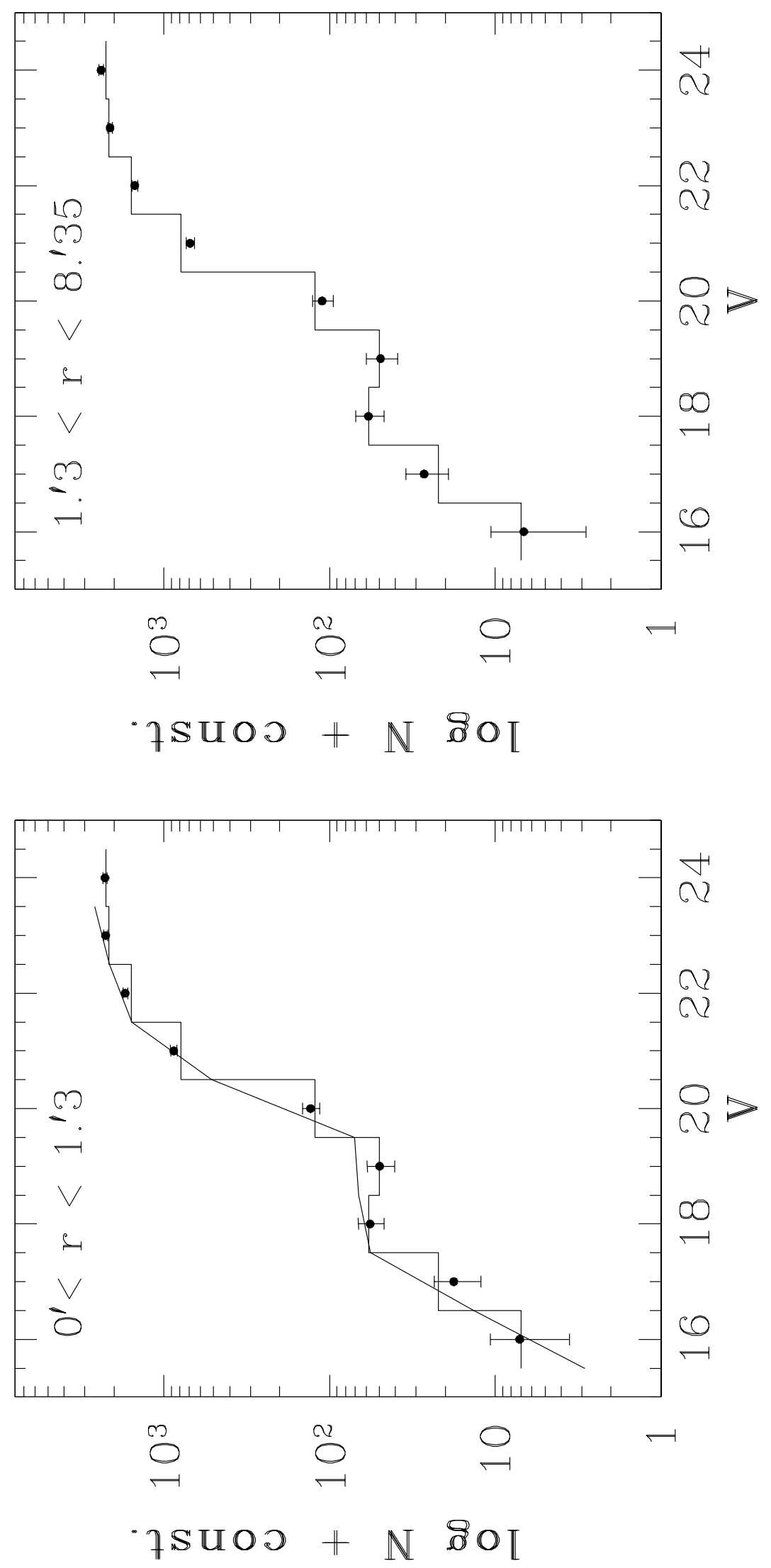


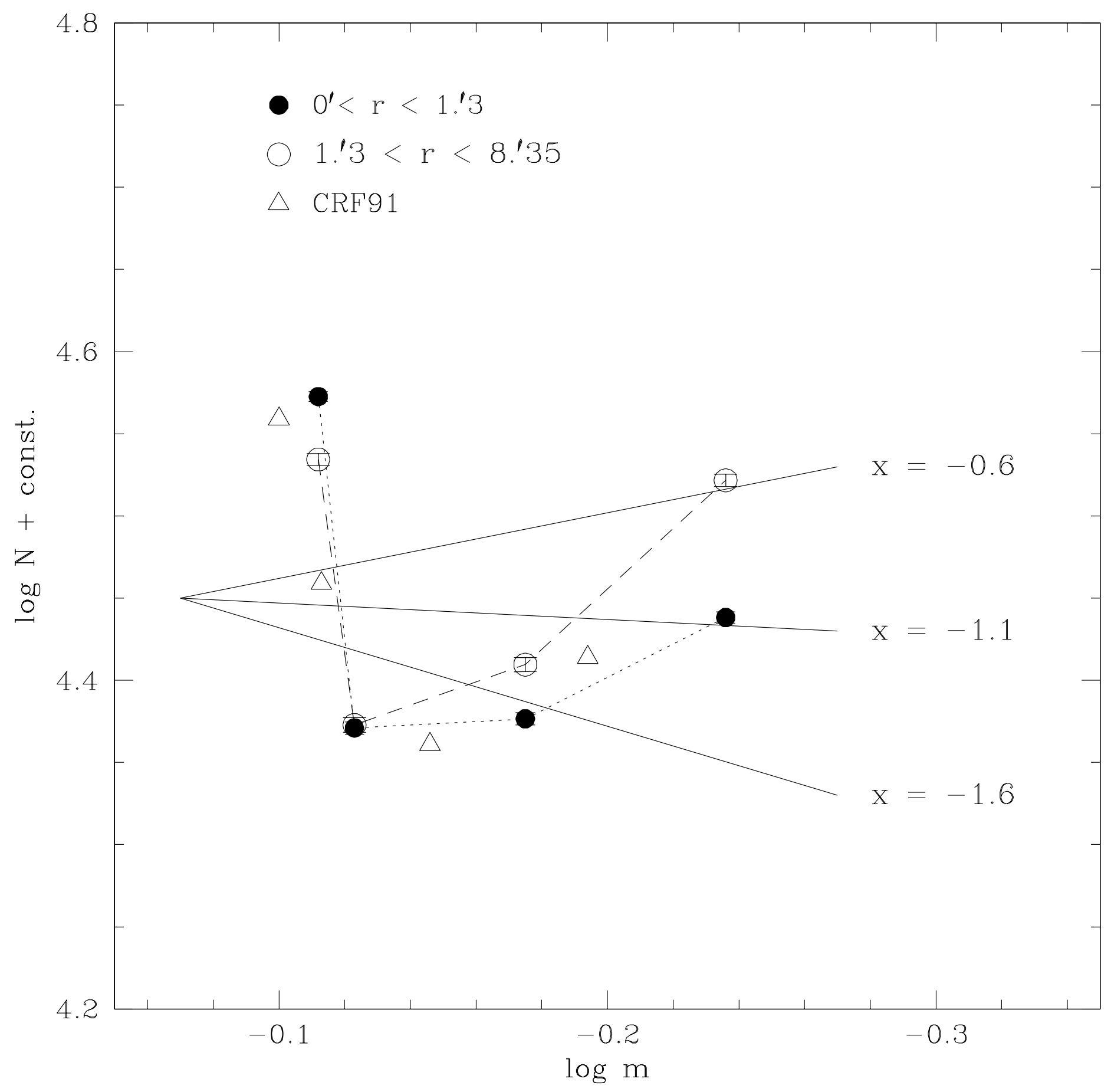


This figure "Lee.fig09.jpg" is available in "jpg" format from: http://arxiv.org/ps/astro-ph/0409037v1 


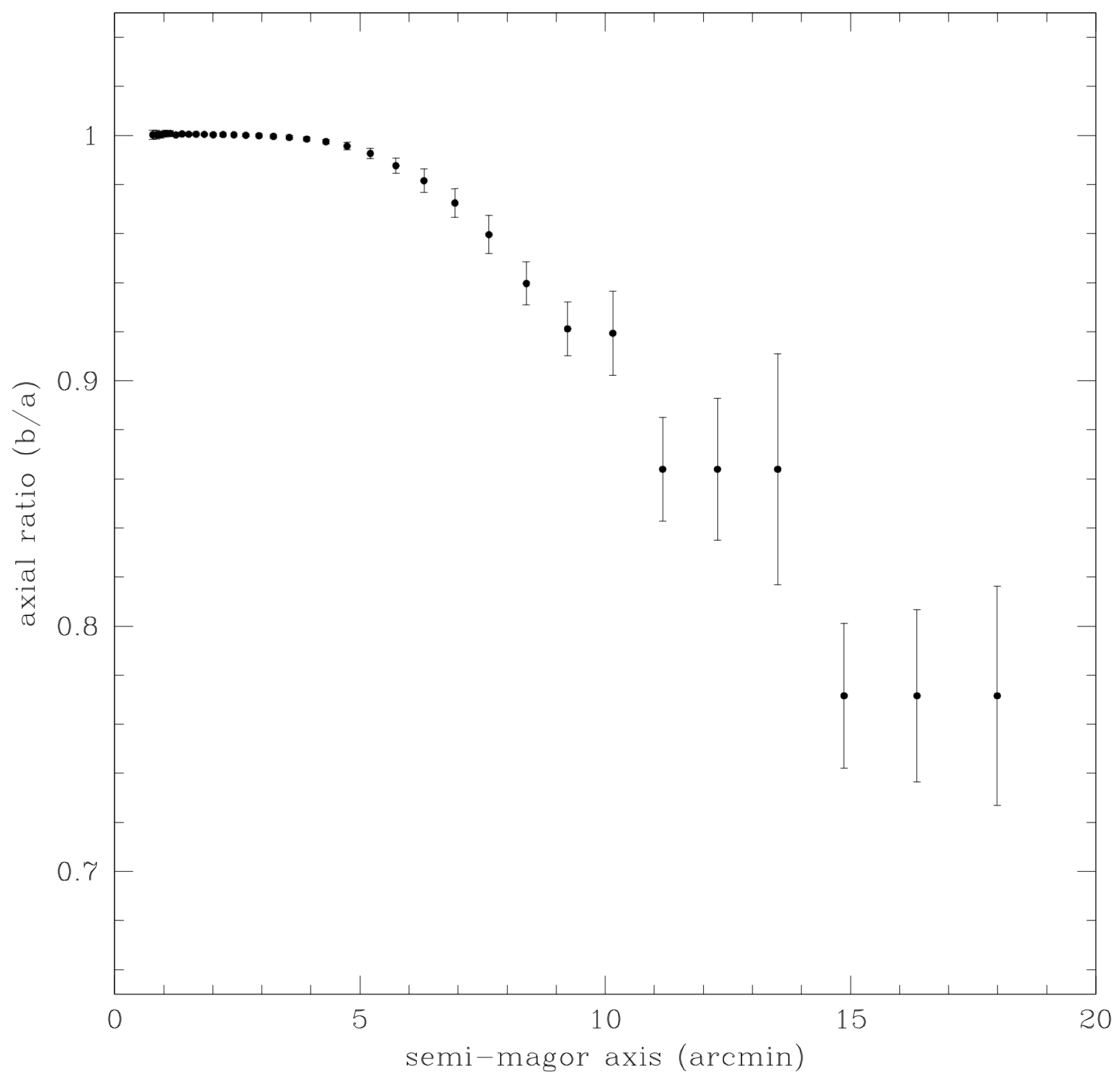




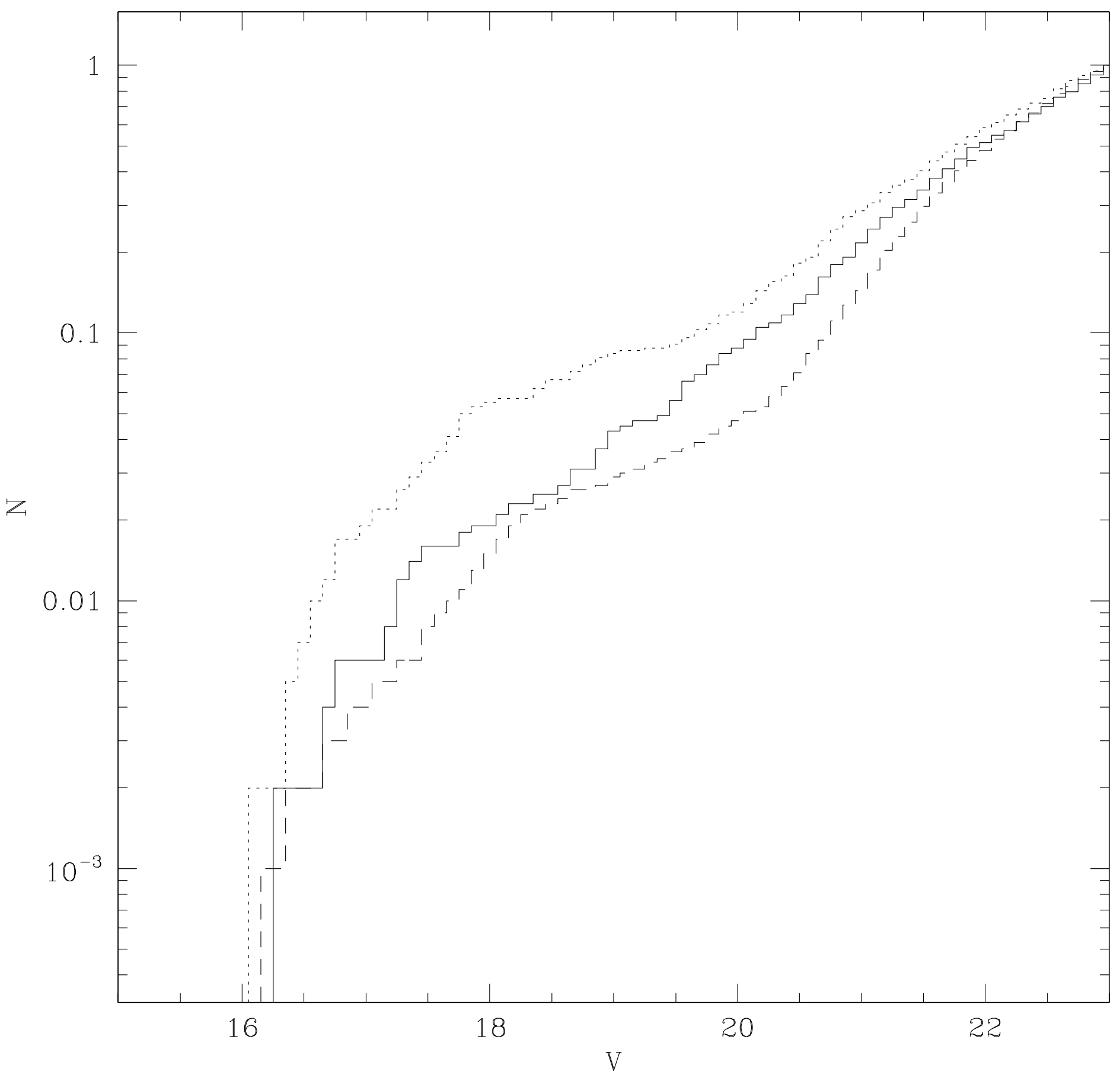

